Revue d'histoire de l'Amérique française

REYUE D.HISTOIRE DE L'AMÉRIQUE FRANÇAISE

\title{
Le Canada et les hommes politiques de 1867
}

\section{Jean-Charles Bonenfant}

Volume 21, numéro 3a, 1967

Cent ans d'histoire 1867-1967

URI : https://id.erudit.org/iderudit/302713ar

DOI : https://doi.org/10.7202/302713ar

Aller au sommaire du numéro

Éditeur(s)

Institut d'histoire de l'Amérique française

ISSN

0035-2357 (imprimé)

1492-1383 (numérique)

Découvrir la revue

Citer cet article

Bonenfant, J.-C. (1967). Le Canada et les hommes politiques de 1867. Revue d'histoire de l'Amérique française, 21(3a), 571-596.

https://doi.org/10.7202/302713ar d'utilisation que vous pouvez consulter en ligne.

https://apropos.erudit.org/fr/usagers/politique-dutilisation/ 
Jean-Charles Bonenfant

\author{
LE CANADA \\ ET LES HOMMES \\ POLITIQUES \\ DE 1867
}




\section{Jean-Charles Bonenfant}

Né le 21 juillet 1912 à Saint-Jean de l'Ile d'Orléans où son père était médecin. Etudes secondaires au Séminaire de Québec. Bachelier ès arts, 1932. Etudes universitaires à la Faculté de Droit, à la Faculté des Lettres et à la Faculté de Philosophie de Laval. Licencié en droit, 1935. Admis au Barreau la même année.

Journaliste à l'Evénement-Journal de Québec 1934-37; secrétaire du Premier Ministre de la province de Québec (1937-39); assistantbibliothécaire à la Législature de la province de Québec (1939-52); directeur de la Bibliothèque de la Législature de la province de Québec depuis 1952; conseiller juridique de l'Assemblée législative du Québec.

Professeur à la Faculté de Droit, à la Faculté des Sciences sociales, à l'Institut d'Histoire et à la Faculté d'Education de l'Université Laval.

Collaborateur à plusieurs publications dont la Revue du Barreau, Culture, Revue de l'Université Laval, le Bulletin de l'Association canadienne des bibliothèques, la Revue du Notariat, Toronto University Quarterly, Canadian Annual Review. Auteur d'un livre sur les institutions politiques canadiennes.

Membre de la Sociécé royale du Canada, de la Société des Dix, de l'Association canadienne des bibliothécaires de langue française, de l'Association canadienne des bibliothèques, de l'Association canadienne d'Histoire, membre du Barreau de Québec.

En 1955 et en 1966, a donné, sous les auspices de l'Institut scientifique franco-canadien, des cours dans les universités françaises. à 1967.

Président de la Commission de géographie de Québec de 1962

Marié et père de quatre enfants.

Résidence: 1450, av. du Buisson, Sillery. 


\section{LE CANADA ET LES HOMMES POLITIQUES DE 1867}

L'histoire n'est sans doute pas uniquement, comme le prétendait naguère Carlyle, celle des "grands hommes qui ont travaillé ici-bas" et nous savons aujourd'hui qu'une foule d'inconnus aussi bien que des forces obscures ont engendré les événements, mais il reste qu'à certains moments des hommes sont intervenus pour en précipiter le rythme. Les colonies britanniques en Amérique du Nord étaient sans doute destinées à se réunir un jour en un système fédératif ou unitaire avant la fin du dix-neuvième siècle, mais un certain nombre d'hommes politiques en Amérique et en Grande-Bretagne ont suffisamment influencé les événements qui se sont déroulés de 1860 à 1868 pour qu'il convienne d'en rappeler le souvenir. Les plus connus de ces hommes sont évidemment les trente-trois Pères de la Confédération tels que les a fait revivre le tableau de Robert Harris popularisé par l'imagerie populaire. ${ }^{1}$

1 Rappelons que le tableau de Robert Harris, peintre canadien né au pays de Galles en 1849, venu très jeune dans l'Ile-du-Prince-Edouard, et qui mourut en 1919, n'a pas été peint sur les lieux à Québec en octobre 1864, mais a été commencé plus tard en 1883. Le 14 mai 1883, Wilfrid Laurier, qui n'était pas encore chef du parti libéral, mais simple député, ancien ministre et membre de l'opposition, attira l'attention de la Chambre des communes sur un mémoire qui avait été présenté au gouvernement conservateur de John A. Macdonald par l'Académie royale des arts du Canada. Le mémoire disait en résumé que la Conférence au cours de laquelle avaient été étahlies les bases de la Confédération était un événement d'une telle importance dans les annales du pays qu'il était désirable de le rappeler dans un tableau qui représenterait les portraits authentiques des hommes d'état qui avaient pris part aux délibérations. Le mémoire faisait remarquer que chaque année qui s'écoulait ajoutait aux difficultés de faire ce tableau, car déjà plusieurs des personnages qui devaient y figurer étaient morts. Enfin, l'Académie déclarait ceci: "Une occasion s'offre maintenant pour l'exécution de ce travail par la présence en Canada de M. Harris, un artiste canadien de grands talents qui vient de terminer ses études et pratiquer son art en Europe et qui est tout à fait compétent pour faire un tableau de ce genre." 
Il faut y ajouter trois hommes politiques auxquels, en 1927, on a reconnu le titre de Pères de la Confédération parce qu'ils avaient participé à la Conférence de Londres: William Pearce Howland, du Canada-Uni, John Wm. Ritchie, de la NouvelleEcosse, et Robert D. Wilmot, du Nouveau-Brunswick. On pourrait donc tracer une sorte de hiérarchie des Pères de la Confédération. Il y a d'abord ceux qui ne participèrent qu'à la Conférence de Londres et que nous venons de nommer. Howland n'était entré dans le cabinet de coalition du Canada-Uni, dirigé par Etienne-Paschal Taché, qu'après la Conférence de Québec en novembre 1864, pour y remplacer Oliver Mowat devenu juge. Il refusa de suivre George Brown lorsque ce dernier quitta le cabinet en 1865 et, en 1866, il devint ministre des Finances à la démission d'Alexander T. Galt. John Wm. Ritchie, solliciteur général de la Nouvelle-Écosse, de 1864 à 1867, n'était pas à la Conférence de Québec en octobre 1864, mais il représentait sa colonie à une autre Conférence de Québec, celle de septembre

\footnotetext{
La commande fut donnée à Harris pour une somme de dix mille dollars. Le peintre se mit à l'œuvre et il reconstitua une séance de la Conférence. En 1884, le tableau des Pères de la Confédération fut placé dans le grand hall des édifices parlementaires à Ottawa où il brûla lors de l'incendie de février 1916. Trop âgé pour refaire le tableau, Harris se contenta d'en retoucher l'esquisse au fusain pour l'offrir au gouvernement. Elle est aujourd'hui dans la salle du comité des chemins de fer. Le tableau a valu à son auteur une accusation de plagiat. On prétendit que ce n'était qu' "une vulgaire imitation" d'un tableau de Thomas Faed fait, en 1850, et représentant Walter Scott et ses amis à Abbotsford. Scott étant mort en 1832, le tableau de Faed était donc posthume comme celui de Harris. "Le peintre qui a fait le tableau des Pères de la Confédération, écrivait, dans le Bulletin des Recherches historiques de 1896 (p. 122), le notaire historien JosephEdmond Roy, a tout copié: jeu de lumière, mise en scène, disposition des personnages. L'imitation est si servile qu'elle frappe à première vue." On se montrait surpris que John A. Macdonald, en bon Ecossais admirateur de Walter Scott, n'ait pas noté et dénoncé la ressemblance. Notons qu'il y a dans le tableau trente-quatre personnages alors qu'il n'y avait à la Conférence de Québec que trente-trois représentants des colonies. Le trente-quatrième personnage qui s'estompe discrètement à gauche du tableau est Hewitt Bernard (1825-1893) qui de 1858 à 1866 fut secrétaire de John A. Macdonald, devint en 1867 son beau-frère, assista à la Conférence de Charlottetown, et fut secrétaire de la Conférence de Québec et de la Conférence de Londres. Il fut plus tard le premier sous-ministre de la Justice sous la Confédération. On lui doit une source importante de renseignements sur la naissance de la Confédération car ce sont ses notes qui forment en grande partie le recueil tiré des archives de John A. Macdonald et publié sous le titre de Pope's Confederation Documents (Toronto, 1895).
} 
1865, sur la négociation des traités commerciaux. ${ }^{2}$ Robert $\mathrm{D}$. Wilmot, représentait le Nouveau-Brunswick à cette dernière Conférence et il semble s'y être converti à l'idée d'un système fédératif auquel il s'était opposé jusque-là. En 1866, il abandonna le parti des adversaires de la Confédération pour entrer dans le gouvernement de Peter Mitchell en qualité de ministre sans portefeuille. Il accompagna le premier ministre à Londres.

Il y a ensuite les huit hommes qui n'assistèrent qu'à la Conférence de Québec: les deux délégués de Terre-Neuve, Frederick Bowker T. Carter et Ambrose Shea, qui d'ailleurs ne furent plutôt que des observateurs; Thomas $H$. Haviland et Edward Whelan, de l'Île-clu-Prince-Edouard; Étienne-Paschal Taché, Jean-Charles Chapais, James Cockburn et Oliver Mowat, du Canada-Uni. Furent présents à Charlottetown et à Québec les douze suivants: Thomas d'Arcy McGee, Alexander Campbell, George Brown, du Canada-Uni; John Hamilton Gray, Edward Palmer, Andrew A. Macdonald, William H. Pope et George H. Coles, de l'Île-du-Prince-Edouard; William H. Steeves, John Hamilton Gray, Edward B. Chandler, du Nouveau-Brunswick; Robert B. Dickey, de la Nouvelle-Écosse. Peter Mitchell et Charles Fisher, du Nouveau-Brunswick, étaient à Québec et à Londres.

Enfin, il y a les onze hommes qui participèrent aux trois conférences et auxquels il faut ajouter le nom du secrétaire Hewitt Bernard. Il n'y manque que le nom de George Brown pour qu'on puisse affirmer que ce sont, sauf dans quelques cas, vraiment les vedettes: John A. Macdonald, George-Étienne Cartier, Hector-L. Langevin, William McDougall et Alexander T. Galt, du Canada-Uni ; Charles Tupper, William A. Henry, Adams G. Archibald et Jonathan McCully, de la Nouvelle-Écosse; S. Leonard Tilley et John M. Johnson, du Nouveau-Brunswick.

Il ne suffit pas de s'arrêter aux "Pères de la Confédération" lorsqu'on veut parler des hommes politiques qui ont été mêlés à la naissance du régime fédératif, mais il convient d'y ajouter

2 Cette réunion est moins connue et eut moins d'importance que celle d'octobre 1864, mais certains historiens y attachent une certaine importance. Cf. N. McL. Rogers, "The Confederate Council of Trade", Canadian Historical Review, VII, 4 (Dec., 1926) : 277-286. 
les adversaires du projet et les personnages qui, en GrandeBretagne, le favorisèrent. Parmi les noms qui mériteraient donc encore d'être retenus, il y aurait dans les colonies: AntoineAimé Dorion, Jean-Baptiste-Éric Dorion, Christopher Dunkin, Luther Hamilton Holton, Lucius Seth Huntington, John Sandfield Macdonald, dans le Canada-Uni et Joseph Howe, en Nouvelle-Écosse. J'ajouterais volontiers le nom d'un simple député qui, lui, fut favorable à la Confédération: Joseph-Édouard Cauchon. Chez les hommes politiques de la métropole, je nommerais lord Monck, qui en sa qualité de gouverneur des colonies britanniques en Amérique du Nord fut un trait d'union officiel entre Londres et les colonies, Edward W. Watkin, qui lui fut un trait d'union officieux, le duc de Newcastle, Edward Cardwell, C. B. Adderley et lord Carnarvon.

Quelque cinquante hommes politiques ont donc été mêlés aux événements qui se sont déroulés de 1864 à 1867 et même si une dizaine s'en détachent et méritent qu'on leur donne plus d'importance, je tenterai dans un rapide tour d'horizon de montrer ce que fut l'activité du plus grand nombre.

\section{LES PERES DE LA CONFEDERATION}

Canadien français et écrivant dans une revue canadiennefrançaise, je voudrais d'abord m'arrêter aux quatre "Pères de la Confédération" de langue française un peu écrasés par tous leurs collègues anglo-saxons. Officiellement, le premier d'entre eux, c'est Étienne-Paschal Taché, "Sir Etienne", depuis 1858, alors qu'il avait été investi chevalier par la reine Victoria ellemême au château de Windsor. Né en 1795 , c'est un vieillard lorsqu'en mars 1864, il accepte de sortir de sa retraite pour devenir premier ministre théorique d'un cabinet conservateur avec John A. Macdonald. Très loyaliste, en 1812, alors qu'il n'était qu'un adolescent, il s'était battu pendant la guerre de 1812. Il avait ensuite étudié la médecine à Québec et à Philadelphie pour aller exercer sa profession dans sa paroisse natale de Saint-Thomas de Montmagny. Elu député en 1841, lors des premières élections de l'Union, il avait poursuivi une heureuse 
carrière politique qui le fit ministre et qu'il interrompit de 1846 à 1848 pour s'occuper de la milice du Bas-Canada. En 1857, il s'était retiré de la politique active tout en demeurant conseiller législatif. C'est lui qui, en 1846, d'après La Minerve du 27 avril, aurait lancé cette prophétie grandiloquente qui fait sourire aujourd'hui : "Si jamais ce pays cesse un jour d'être britannique, le dernier coup de canon tiré pour le maintien de la puissance anglaise en Amérique le sera par un bas Canadien." Il devint, en juin 1864, comme une sorte de patriarche autour duquel se fit l'unanimité, premier ministre théorique du gouvernement de coalition, et c'est à ce titre qu'il fut président de la Conférence de Québec, rôle qu'il remplit à la satisfaction de tous, mais qui l'épuisa. Il ne devait pas voir la naissance de la Confédération car il mourut le 30 juillet 1865 . La Confédération aurait pu naître sans Étienne-Paschal Taché et ce n'est pas lui, malgré son titre de premier ministre, qui était le véritable chef des Canadiens français, mais c'était un homme digne, ayant un sens profond de ses devoirs et il est heureux qu'il ait été mêlé aux événements de 1864. Il contribua en particulier à maintenir l'harmonie dans le cabinet de coalition. Il est d'usage de prétendre que l'histoire n'a pas rendu justice à Taché. Un membre de sa famille, le R.P. Louis Taché, a même terminé l'intéressant article qu'il lui a consacré par la réflexion que si Brown et Cartier avaient immolé des ressentiments personnels, "Sir Etienne Taché a sacrifié un bien plus grand, celui auquel on tient par-dessus tout: la vie." 3 Mais l'histoire se rappelle surtout ceux qui détiennent vraiment le pouvoir et dont l'action peut directement influencer le cours des événements et cet homme ce fut non pas Taché, mais George-Etienne Cartier.

Aussi sent-on à peine le besoin de parler des deux autres Pères de la Confédération canadiens-français qui, si estimables qu'ils aient pu être, ne pouvaient que se taire lorsqu'un chef autoritaire comme Cartier était là. Hector Langevin, né à Québec en 1826, était un des plus jeunes parmi les "Pères de la Confédération". Il avait fait ses études au Petit Séminaire de Québec,

${ }^{8}$ Louis Taché "Sir Etienne-Pascal Taché et la Confédération canadienne", Revue de l'Université d'Ottawa (1935): 231-255. 
mais il avait été formé comme avocat à Montréal dans le bureau de Cartier lui-même. Député depuis 1857 du comté de Dorchester, il était entré dans le gouvernement de coalition. Il était le frère d'un membre éminent du clergé qui, en 1867, allait devenir le premier évêque de Rimouski, parenté qui constituait un des nombreux liens entre l'église catholique et le parti conservateur. On sent chez lui, surtout à la Conférence de Londres, l'impatience du cadet en face d'un aîné qui s'attribue tous les mérites. Langevin a connu après la Confédération une longue carrière politique au cours de laquelle il ne réussit jamais à remplacer Cartier comme chef des Canadiens français et qui se termina en 1891 par un scandale pour lequel il fut toutefois exonéré. Il se retira de la vie publique et mourut en 1906.

Le quatrième Canadien français "Père de la Confédération" fut Jean-Charles Chapais, né en 1811 et qui avait succédé comme marchand à son père après des études secondaires. Depuis 1851, il était député de Kamouraska et, depuis 1864, ministre des Travaux publics. C'était un bon politicien qui fut mêlé à des luttes locales violentes. C'était aussi un administrateur consciencieux. Il ne semble pas avoir pris une part très active à la Conférence de Québec, la seule réunion à laquelle il assista. Mlle Julienne Barnard qui, sous le titre de Mémoires Chapais, a scruté avec méthode et piété tout ce qui a trait à Jean-Charles Chapais ne peut qu'affirmer: "Un article biographique, écrit à la mémoire de Jean-Charles Chapais dans le Courrier $d u$ Canada (numéro du 18 juillet 1885) affirme qu'il prit une part active aux délibérations, mais dans quelle mesure ?... Il ne se peut guère qu'il soit resté passif, quand on connaît son passé politique, ses connaissances législatives et son tempérament." ${ }_{4}$ C'est évidemment assez mince comme témoignage. Chapais devait mourir sénateur en 1885.

Le seul chef des Canadiens français, celui qui prendra les véritables décisions, c'est George-Étienne Cartier, George sans "s" à la manière anglaise, et il y tenait, car il prétendait avoir reçu ce prénom en l'honneur du roi d'Angleterre, George III. ${ }^{5}$

4 Julienne Barnard. Mémoires Chapais (3 vol.. Fides, 1966), 2: 242.

5 Benjamin Sulte, Mélanges historiques, 4 (1919) : 88. 
En 1864, il a cinquante ans. Reçu avocat en 1855 , il a pris part aux troubles de 1837 avant de s'enfuir aux États-Unis. Bien rangé, il aime dire avec une certaine coquetterie à ses amis anglais: “Quand j'étais un rebel !" Député depuis 1848, ministre une première fois en 1856, il a édifié avec John A. Macdonald le puissant parti conservateur. A compter de 1857, il a été l'artisan de nombreuses réformes et il a réussi en particulier à faire effectuer d'importantes codifications. Il se convertit à l'idée du fédéralisme en 1858, lors de l'entrée d'Alexander T. Galt dans le cabinet qu'il dirige avec John A. Macdonald. Il comprend alors que si l'Union, édictée contre les Canadiens français, s'est avec les années transformée en un régime qui leur est favorable, que si le Bas-Canada dont la population est devenue moindre que celle du Haut-Canada a quand même la moitié des représentants à l'Assemblée législative et que si l'aile conservatrice québécoise qu'il dirige est le groupe le plus influent de la Chambre, ces avantages ne pourront durer indéfiniment et qu'un jour il faudra bien accepter la représentation selon la population réclamée par le Haut-Canada. Il s'y est toujours opposé, mais il juge qu'elle n'aurait pas les mêmes inconvénients dans un état fédératif où, croit-il, l'indépendance naturelle des provinces maritimes s'alliera aux intérêts ethniques du Bas-Canada pour s'opposer au Haut-Canada, d'autant plus que la future province du Québec aura sa législature maîtresse de plusieurs compétences importantes. Par ailleurs, Cartier craint presque maladivement les ]États-Unis. "La question, déclare-t-il le 7 février 1865, dans le grand discours qu'il prononce à l'Assemblée législative en faveur des Résolutions de Québec, se réduit à ceci: il nous faut ou avoir une confédération de l'Amérique britannique du Nord, ou bien être absorbés par la confédération américaine." Il est en outre lié aux intérêts ferroviaires qui désirent la Confédération et il est naturel que comme tout homme politique il souhaite jouer un rôle sur une scène plus étendue. Ce n'est pas un théoricien et sa conception du fédéralisme est assez pragmatique. Il fait tout ce qu'il peut pour sauvegarder les intérêts des Canadiens de langue française, mais seuls à ses 413-414.

6 Discours de Sir Georges Cartier par Joseph Tassé (Montréal, 1893). 
yeux comptent ceux qui habitent le Bas-Canada. Il semble s'être défié jusqu'à un certain point de John A. Macdonald. D'après des documents, qui n'offrent cependant pas une certitude historique absolue, pendant la Conférence de Londres, à la fin de 1866, John A. Macdonald aurait essayé de tromper son collègue Cartier en faisant décréter une sorte d'union législative plutôt que la Confédération qui avait été décidée à Québec. Cartier aurait menacé de le dénoncer et tout serait entré dans l'ordre. ${ }^{\top}$ Quoi qu'il en soit, on peut conclure avec cette affirmation d'un journaliste historien à ses heures, sir John Willison, "sans Cartier et le clergé catholique du Québec, l'Union de 1867 n'aurait pu s'accomplir "". Par ailleurs, il est injuste de le rendre responsable des insuccès d'un régime qu'il ne pouvait vraiment pas prévoir à une époque où le meilleur gouvernement était celui qui intervenait le moins possible dans la vie de ses habitants et à une époque où les minorités attachaient plus d'importance à la religion qu'à la langue.

Le grand artisan de la Confédération fut John A. Macdonald qui a laissé dans notre histoire politique une réputation légendaire. Veuf depuis quelques années, en 1864, il approchait de la cinquantaine et il était dans toute la force de son habileté. C'était un joyeux vivant pour ne pas dire plus. Il était né en Écosse, mais très jeune il était venu avec ses parents dans le Haut-Canada. Il était avocat, mais la politique l'avait plus occupé que le droit. Il ne croyait pas énormément à la Confédération et aurait été plutôt en faveur d'une union législative, mais dès qu'il s'aperçut qu'un système fédératif était le seul possible il s'en fit le meilleur des apôtres. C'est lui qui fut l'âme de la coalition de juin 1864 et le grand artisan de la Conférence de Québec dont il a rédigé une bonne partie des propositions en raison de la procédure suivante. En général, avant chaque séance de la Conférence, il y avait une réunion du cabinet du Canada-Uni, au cours de laquelle on préparait des propositions

7 Cf. à ce sujet les documents publiés par l'abbé Lionel Groulx en appendice à son livre La Confédération canadienne (Montréal, 1918). 1903), 1: 91 . 
qui étaient ensuite soumises à la Conférence et il était alors naturel que Macdonald devint le porte-parole du cabinet. Signalons en parenthèse que cette procédure expliquerait le fait que Cartier ne semble guère avoir parlé pendant la Conférence, du moins d'après les documents incomplets que nous possédons. C'est au sein du Cabinet que l'homme d'état canadien-français aurait fait valoir les droits de ses compatriotes. ${ }^{\theta}$ Macdonald continuera à jouer le rôle le plus important de la politique canadienne, de 1867 jusqu'à sa mort en 1891, sauf pendant les quelques années qu'il passa dans l'opposition à la suite du scandale du Pacifique. Son nom remplit notre histoire pendant la seconde partie du XIX siècle et c'est sous son règne que le Canada est devenu un pays véritable en s'étendant de l'Atlantique au Pacifique. Par réalisme plutôt que par générosité, il a été un des premiers hommes d'état anglo-saxons à accepter les Canadiens français comme des partenaires égaux, mais par ailleurs lors de la condamnation de Louis Riel, en 1885, il a brisé l'unité canadienne en refusant que le chef métis soit gracié parce qu'à ce moment il a cru que les protestations de l'Ontario étaient plus importantes que celles du Québec.

Après Macdonald, il faut nommer George Brown, le chef des libéraux canadiens-anglais du Haut-Canada. Avec Cartier, c'est l'homme qui a rendu possible la Confédération parce qu'il représentait en quelque sorte le second pôle d'un axe nécessaire à l'entente. C'était le chef des éléments du Haut-Canada sincèrement opposés aux Canacliens français et aux "Papistes" dont il redoutait vraiment la domination. Fondateur et propriétaire du journal le Globe de Toronto, il détestait Macdonald, mais il sut surmonter bien des préjugés pour entrer, en 1864, dans le gouvernement de coalition qui assura la naissance de la Confédération. Pour comprendre l'importance de l'événement, il suffit de lire le portrait que le journal conservateur $L a$ Minerve traçait le 21 juin du nouvel allié. "Il a fait appel, écrit-on, aux préjugés et aux animosités de race, au fanatisme et à l'intolérance religieuse. Il a dépeint les Bas-Canadiens sous les cou-

\footnotetext{
$9 \mathrm{~W}$. Menzies Whitelaw, "Reconstructing the Quebec Conference", The Canadian Historical Review, 19: 123-137.
} 
leurs les plus noires, comme une population ignorante, routinière, superstitieuse et traînant une existence pauvre et misérable, sous contrôle absolu et tyrannique du clergé:" Évidemment pour La Minerve, Brown avait changé et en quelque sorte il se convertissait en acceptant d'entrer dans la coalition. Et le journal ajoutait, avec une certaine grandiloquence: "Eh bien ! franchement, la main sur la conscience, malgré l'aversion bien naturelle que nous avons eue jusqu'ici pour M. Brown, nous n'hésitons pas à reconnaître qu'il a fait là un acte vraiment grand et admirable et qui rachète bien des erreurs de son passé. Ils déposeront au pied de l'autel de la patrie où le grand prêtre $\mathrm{du}$ "cleargritisme" vient de consommer le plus douloureux des sacrifices, leurs colères et leurs ressentiments légitimes." Brown sortit de la coalition à l'automne de 1865 , mais il resta favorable à la Confédération. Après le 1er juillet 1867, il redevint un libéral farouche. Il mourut en 1880 assassiné stupidement par un employé qu'il avait congédié.

Alexander T. Galt, député de Sherbrooke, chef de la minorité anglo-saxonne et protestante du Bas-Canada, était né en Grande-Bretagne et était venu très jeune au Canada où il avait participé aux immenses spéculations immobilières de sa famille. En 1849, il avait signé comme bien d'autres de ses compatriotes un manifeste en faveur de l'annexion aux Etats-Unis qu'on lui rappela parfois. En 1858, il fut le premier homme politique à proposer sérieusement un système fédératif et il en fit la condition de son entrée dans le gouvernement. Il fut plus tard l'inspirateur des dispositions financières du projet élaboré à la Conférence de Québec, mais pour les Canadiens de langue française l'aspect le plus intéressant de son activité fut sans doute le soin qu'il apporta à protéger dans la future province de Québec les droits de la minorité anglo-saxonne. Celle-ci ne voulut prendre aucun risque. Elle représentait près du quart de la population du Bas-Canada, mais sa puissance réelle était plus considérable que sa force numérique, car dans les villes de Québec et de Montréal, elle était maîtresse de l'industrie, du commerce et des finances et elle dominait presque entièrement les Cantons de l'Est. Elle craignait que la nouvelle constitution ne lui fasse 
perdre sa situation privilégiée, soumise qu'elle serait dorénavant dans une foule de domaines au bon vouloir d'une majorité canadienne-française et catholique au sein de la future législature locale. Galt tenta de rassurer ses compatriotes dans un grand discours qu'il prononça le 23 novembre 1864, dans la ville de Sherbrooke.10 "Si les Canadiens français, déclara-t-il, étaient assez insensés pour s'attaquer à nos intérêts particuliers, la rétribution ne se ferait pas longtemps attendre, soit dans la législature générale, soit mème dans la législature locale. Mais cela n'arrivera point. Nous continuerons à vivre unis et heureux, comme nous vivons aujourd'hui dans cette bonne ville de Sherbrooke. Mais en même temps, il ne faut pas négliger les moyens constitutionnels de prévenir tout empiétement d'une race contre l'autre." 11 Galt repoussa l'idée que les Canadiens français puissent un jour priver leurs compatriotes anglais d'une représentation substantielle à l'Assemblée législative, mais il ne se fia pas à leur générosité. Il exigea dans le projet de la future constitution du Québec, présentée en 1866 au Parlement du CanadaUni, qu'aucune modification ne pût être apportée aux bornes d'une circonscription électorale sans l'approbation des trois quarts des députés, soit 49 sur 65 , ce qui aurait exigé le consentement des députés anglais. La disposition se transforma quelque peu pour devenir l'article 80 de l'Acte de l'Amérique du Nord britannique. ${ }^{12}$ La minorité anglo-saxonne du Bas-Canada fut en outre une des causes de la création du Conseil législatif du Québec. Galt avait aussi promis à ses compatriotes que différents changements seraient faits dans les lois d'éducation du Bas-Canada, dans l'intérêt de la minorité protestante. Il ne réussit pas au cours de la session de 1866 à faire adopter une loi à cet effet mais, quoique ne faisant plus partie du cabinet canadien, il participa à la Conférence de Londres où il s'occupa des intérêts de ses coreligionnaires. Il y fit rédiger la version

10 Speech on the Proposed Union of the British North American Provinces by the Hon. A. T. Galt (Montreal, 1864). La Minerve publia une traduction assez complète du discours à compter du 28 novembre.

11 La Minerve, 30 novembre 1864.

12 Sur cette question, cf. Jean-Charles Bonenfant, "Les douze circonscriptions électorales privilégiées du Québec", Cahiers de géographie, no 13 (avril-sept., 1962). 
définitive de l'article 93 de l'Acte de l'Amérique du Nord britannique. Trente ans plus tard, à la Chambre des communes, Charles Tupper pouvait déclarer: "On a insisté sur l'insertion de cet article dans l'intérêt non des catholiques mais des protestants. Je dis que nous n'aurions pas eu de Confédération, que tout ce projet eut échoué misérablement si nous avions refusé d'inclure cette protection de la minorité protestante de Québec, telle que représentée par Sir Alexander Galt. Ceux de mes collègues qui étaient présents dans cette occasion ne me démentiront pas si je dis que Sir Alexander Galt était si catégorique sur ce point que jusqu'à ce que la conférence eut adopté cette politique, il ne voulut ni prendre part ni aider en quoi que ce soit à l'accomplissement de la Confédération."18 Galt mourut en 1893 après avoir été premier haut-commissaire du Canada à Londres.

Les Irlandais catholiques étaient assez nombreux dans le Canada-Uni pour être représentés dans le gouvernement et aux conférences de Charlottetown et de Québec. Ils le furent par Thomas d'Arcy McGee, un homme remarquable, orateur éloquent, bon écrivain, le plus Canadien des Pères de la Confédération. Né en Irlande en 1825, il avait été journaliste aux ÉtatsUnis, était retourné dans son pays d'origine pour y faire de l'agitation révolutionnaire. Menacé d'arrestation, il avait réussi, déguisé en prêtre, à s'enfuir aux Etats-Unis pour venir au Canada en 1857 et être élu député dès 1858. D'abord libéral, il passa ensuite au parti conservateur pour entrer dans le gouvernement en 1864. Il comprit mieux que tout autre qu'une nouvelle nation, au sens anglais du mot, naissait. Il transporta son nationalisme irlandais dans un nouveau contexte. Dès 1860 , parlant à la législature du Canada-Uni, il déclarait: "Nous avons fait quelques progrès dans la voie du développement d'une nationalité et la législature si elle peut retarder cette évolution ne peut pas vraiment l'empêcher. Le jour n'est pas lointain où nous ne serons plus des habitants du Haut-Canada, du BasCanada, de la Nouvelle-Écosse ou du Nouveau Brunswick, mais

13 Débats de la Chambre des Communes du Canada (1896), 2406. 
les membres d'une nation embrassant les six colonies unies." 14 Malheureusement, McGee n'assista qu'aux premiers pas de cette nation dont il rêvait, car il mourut, en 1868, assassiné par un Irlandais fanatique qui lui reprochait sa tiédeur.

Mentionnons plus rapidement les autres Pères de la Confédération qui représentèrent le Canada-Uni : deux conservateurs du Haut-Canada, Alexander Campbell, qui avait étudié au collège de Saint-Hyacinthe et qui comme avocat était l'associé professionnel de John A. Macdonald, et James Cockburn, qui, en 1867, allait devenir le premier orateur de la Chambre des communes; deux libéraux aussi du Haut-Canada: William McDougall qui, contrairement à Brown, demeura avec les conservateurs et joua après la Confédération un rôle important dans la marche du Canada vers l'Ouest et Oliver Mowat, qui, après 1867, fut le père de l'autonomie provinciale dans les luttes politiques et juridiques qu'il mena contre John A. Macdonald en qualité de premier ministre d'Ontario.

La vedette du Nouveau-Brunswick fut S. Leonard Tilley. Puritain sincère, abstème militant en faveur de la tempérance, il eut beaucoup de difficulté à faire triompher la Confédération dans sa province où le peuple fut appelé à se prononcer deux fois au sujet du projet. La première fois, en 1865 , les partisans de la Confédération furent défaits, mais ils triomphèrent l'année suivante. Tilley eut pour le seconder, le premier ministre Peter Mitchell qui, à la Conférence de Londres, s'allia à Cartier pour faire triompher un système plus fédératif qu'unitaire, William Henry Steeves, un homme d'affaires, John M. Johnson, John Hamilton Gray, qu'il ne faut pas confondre avec son homonyme de l'Île-du-Prince-Édouard, qui a laissé un récit de la naissance de la Confédération, ${ }^{15}$ Edward B. Chandler, qui, en 1868, devait devenir un des commissaires pour la construction de l'Intercolonial, et Charles Fisher.

\footnotetext{
15 John Hamilton Gray, Confederation or the political and parliamentary history of Canada from the Conference at Qubbec in October, 1864, to the admission of British Columbia in July, 1871, vol. I (Toronto, 1872). Le volume 2 n'a jamais été publié.
} l'auteur).

14 Thompson's Mirror of Parliament, 1860, no 35, 3 (Traduction de 
En Nouvelle-Écosse, le grand artisan de la Confédération fut Charles Tupper, qui, en 1896, deviendra pendant quelques mois premier ministre du Canada et sera, lorsqu'il mourra en 1915, le dernier survivant des Pères de la Confédération. Il éprouva d'énormes difficultés à faire triompher le projet, mais il réussit finalement à vaincre son adversaire Joseph Howe. Tupper avait à ses côtés à Québec deux conservateurs, R. B. Dickey et William Alexander Henry, qui, à Londres, sera un des principaux rédacteurs du texte de l'Acte de l'Amérique britannique du Nord et deux libéraux, Jonathan McCully et A. G. Archibald.

A la Conférence de Québec, les délégués de l'Île-du-PrinceEdouard, qui ne participa pas à la Conférence de Londres, avaient à leur tête le premier ministre conservateur John Hamilton Gray, qui avait présidé la Conférence de Charlottetown. C'était un militaire qui, en 1867, retourna à l'armée lorsque le projet de confédération fut rejeté par l'Île. Il était accompagné à Québec de trois autres conservateurs, W. H. Pope, le père de Joseph Pope qui fut le dernier secrétaire de John A. Macdonald, son biographe, et le premier sous-secrétaire du Canada aux Affaires extérieures. Les deux autres délégués conservateurs étaient T. H. Haviland et Edward Palmer. Ce dernier fut un des participants les plus critiques de la Conférence de Québec et, revenu dans sa province, il s'opposa avec succès à l'approbation de la Confédération que par ailleurs il favorisa en 1873. Les libéraux étaient George Coles, un brasseur, A. A. Macdonald, qui a laissé un récit de la Conférence de Québec ${ }^{16}$ et Edward Whelan, le journaliste dont les indiscrétions ont permis en bonne partie de reconstituer les événements de l'automne de 1864.

A tous ces noms, il faut ajouter ceux des deux délégués de l'île de Terre-Neuve, un conservateur, F. B. T. Carter, et un libéral, Ambrose Shea. Ils ne jouèrent pas dans les délibérations à Québec un rôle très important.

Tous ces personnages étaient dans la force de l'âge et ils avaient presque tous derrière eux une longue carrière politique.

16 Les notes de A. A. Macdonald ont été publiées dans Canadian Historical Review, 1 (1920). 
Ils représentaient tous les partis politiques, sauf l'aile libérale du Bas-Canada qui était derneurée en dehors de la coalition de juin 1864. C'étaient en majorité des avocats auxquels s'ajoutaient deux médecins, quelques journalistes et des hommes d'affaires. Ce n'étaient pas des théoriciens, mais des hommes pratiques, désireux de sortir de difficultés politiques et économiques plutôt immédiates. Ils connaissaient l'expérience américaine, mais ils étaient incapables de disserter sur les théories du fédéralisme. ${ }^{17}$ Sans se prendre au tragique, ils avaient tout de même conscience de l'œuvre importante qu'ils accomplissaient. George Brown nous le laisse soupçonner lorsqu'il écrivait à son épouse le 14 mars 1865: "Would you not like that darling little Maddie (un de ses enfants) should be able, twenty years hence, when we may be gone, to look back with satisfaction to the share her father had in the great events ? for great they are, and history will tell the tale of them." ${ }^{18}$

\section{LES ADVERSAIRES DE LA CONFEDERATION}

Parmi les hommes politiques qui ont été mêlés à la naissance de la Confédération, il ne faut pas oublier ceux qui en furent les adversaires tenaces de juin 1864 jusqu'aux élections du début de l'automne de 1867. Le seul groupe important demeurant en dehors de la coalition en juin 1864 fut celui des libéraux du Bas-Canada, les "Rouges" auxquels il faut ajouter quelques conservateurs canadiens-français qui se détachèrent de leur parti à cette occasion, un conservateur canadien de taille Christopher Dunkin et deux libéraux anglais du Bas-Canada, Luther Hamilton Holton et Lucius Seth Huntington et dans le Haut-Canada le libéral John Sandfield Macdonald.

Les libéraux canadiens-français eurent alors pour les diriger Antoine-Aimé Dorion, un des grands personnages méconnus de notre histoire dont une biographie vraiment sérieuse n'a

${ }^{17}$ Cf. à ce sujet Jean-Charles Bonenfant, "L'idée que les Canadiens français de 1864 pouvaient avoir du fédéralisme", Culture, 25 (1964) : 307 322.

18 Alex. Mackenzie, The Life and Speeches of Hon. George Brown, (Toronto, 1882), 233. 
malheureusement pas encore été écrite. En 1864, Antoine-Aimé Dorion avait 46 ans. Né à Sainte-Anne-de-la-Pérade en 1818, il avait fait ses études secondaires au collège de Nicolet. Reçu avocat en 1842, il avait milité dans le parti rouge et, en 1854, il avait été élu à l'Assemblée législative du Canada-Uni. En 1858, il avait formé avec George Brown un cabinet de courte durée et, en 1863, il avait été avec J. Sandfield Macdonald, premier ministre. Ce fut l'adversaire le plus sérieux de la Confédération qu'il dénonça dès le 7 novembre dans un manifeste adressé à ses électeurs du comté d'Hochelaga.

Antoine-Aimé Dorion a bien résumé ses arguments dans le discours qu'il prononça le 16 février 1865 et qui peut être regardé comme la somme des arguments des adversaires canadiens-français de la Confédération. ${ }^{10}$ Dorion prétendait que les artisans du projet n'apportaient que deux motifs pour le justifier, la solution du problème de la représentation selon la population et la nécessité de mettre le pays sur un meilleur pied de défense, motifs qui, selon lui, n'étaient pas suffisants. Il ajoutait que le remède préconisé était dangereux pour les Canadiens français et il soutenait que les magnats britanniques des chemins de fer, désireux de rétablir l'état de leurs finances, étaient les artisans secrets de la Confédération.

Dorion admettait toutefois qu'il ne serait peut-être pas toujours nécessairement opposé à la Confédération, mais il jugeait le projet prématuré parce que les provinces qu'on allait unir n'avaient pas entre elles des relations commerciales et sociales suffisantes. Libéral sincère, il dénonçait la Chambre haute non élective, le futur sénat, ce qu'il regardait avec raison comme un recul pour le Canada-Uni qui, depuis 1856, avait une Chambre haute élective.

Enfin, Dorion réclamait une consultation populaire sur le projet, argument puissant auquel Macdonald et Cartier ainsi que leurs partisans ne pouvaient répondre sérieusement. AntoineAimé Dorion lutta jusqu'à la fin contre le projet de Confédé-

19 On trouve le texte du discours d'Antoine-Aimé Dorion des pages 248 à 273 des Débats sur la Confédération. 
ration, mais après les premières élections de 1867 il accepta le nouveau régime. En 1873, il fut ministre de la Justice dans le cabinet MacKenzie et en 1874, il devint juge en chef de la Cour du Banc de la Reine du Québec. Il mourut le 31 mai 1891.

C'était un homme beaucoup plus modéré que son frère cadet Jean-Baptiste-Eric Dorion qui était né en 1826 et qui, journaliste, avait fondé en 1848, L'Avenir, organe du parti rouge. En 1862, il avait fondé un autre journal Le Défricheur. ${ }^{20}$

Dans le discours qu'il prononça sur les résolutions de Québec, Eric Dorion n'ajoutait guère aux arguments de son frère, mais il s'exprima d'une façon plus dramatique. Après avoir montré que la coalition de juin et le projet de confédération qui en était sorti n'étaient qu'une manœuvre du parti conservateur pour conserver le pouvoir, il répéta à satiété: "Je m'oppose au projet de Confédération parce que..." “... Je m'oppose au projet de Confédération parce que ..." 21

Un des derniers actes d'Éric Dorion fut de signer le Manifeste contre la Confédération qui fut publié à la fin d'octobre 1866. En effet, il mourut subitement le 1er novembre 1866, à l'âge de 40 ans.

Les trois députés canadiens-anglais Holton, Huntington et Dunkin étaient dans une situation spéciale puisque la plupart de leurs collègues de langue anglaise du Bas et du Haut-Canada y étaient favorables et qu'ils n'avaient pas à craindre pour la survivance de leur groupe ethnique. Au fond, les deux premiers, libéraux, étaient dépités d'avoir été abandonnés par Brown, par les libéraux du Haut-Canada. Par ailleurs, ils n'étaient pas très sûrs que les intérêts des Canadiens anglais fussent vraiment protégés, comme le prétendait le représentant de ces derniers dans le gouvernement Alexander T. Galt. Huntington déclara même que dans "les townships de l'est", les masses étaient contre le projet, ce qui était exagéré, mais il était plus juste lorsqu'il

\footnotetext{
${ }^{20}$ Sur Jean-Baptiste-Eric Dorion, cf. J.-C. Saint-Amant dans le Bul letin des recherches historiques (1899): 119-123, et Un coin des Cantons de lEst, 257-324.

21 Débats, 859-861.
} 
affirmait qu'en général "les habitants de ces cantons qui appartiennent au parti libéral sont opposés au projet par rapport à la manière dont on l'a présenté au peuple ${ }^{22 "}$.

La position de Christopher Dunkin était plus complexe. Homme remarquable, collaborateur de lord Durham dans sa fameuse enquête, conservateur, premier trésorier provincial du Québec après la Confédération et ensuite ministre de l'Agriculture à Ottawa, il fut un adversaire subtil de la Confédération. Pour expliquer son attitude, il lui fallut plusieurs heures, ce qui lui fit prononcer le plus long discours du débat, soixante-trois pages dans le compte rendu, donc plus de cinq heures. Pour Dunkin, la seule question était: "Comment le projet, dans son ensemble, fonctionnera-t-il ?" Et il l'examinait dans le détail pour trouver mille difficultés, pour se moquer par exemple à une époque où on ne pouvait imaginer la divisibilité de la Couronne de l'offre ... faite... à sa Très Gracieuse Majesté de continuer de la garder sur le trône, en d'autres termes, de la créer Reine de l'Amérique britannique du Nord, par la grâce de la Conférence de Québec. Et Dunkin terminait par cet argument bizarre: "Si l'adoption de ce projet doit nous ramener à l'âge d'or, et rendre nos hommes publics si sages, si prudents et si consciencieux, pourquoi désespérer d'une amélioration dans ce sens lors même que le projet serait rejeté ? Si nous sommes capables de faire fonctionner cette constitution nouvelle et presque impraticable, pourquoi ne serions-nous pas en état de nous en passer ?" 23

A ces noms il faut ajouter celui de John Sandfield Macdonald, catholique du Haut-Canada, libéral, mais ne s'accordant pas avec George Brown. Premier ministre sous l'Union, mais n'étant pas entré dans la coalition de juin, il s'opposa à la Confédération. Les interventions lors du débat de l'hiver de $\mathbf{1 8 6 5}$ sur les Résolutions de Québec furent assez pauvres. Il se rallia rapidement au nouveau régime et à la demande de John A. Macdonald, il devint même en juillet 1867 le premier ministre d'Ontario. 
Le plus illustre adversaire de la Confédération fut sans doute Joseph Howe. Il avait été pendant des années le centre de la vie politique de la Nouvelle-Écosse: il avait conquis la responsabilité ministérielle; il s'était prononcé en faveur de la Confédération, mais devenu, en 1863, fonctionnaire impérial, il resta étranger aux événements qui se déroulèrent depuis 1864 à la fin de 1866. Ayant abandonné son poste de fonctionnaire, il prit la tête du mouvement anti-confédérationiste en NouvelleÉcosse. Il se rendit à Londres pour s'opposer au projet mais John A. Macdonald réussit à le convertir et même à le faire entrer dans son cabinet en 1868.

Enfin, pour compléter le tableau des principaux hommes politiques canadiens qui ont été mêlés à la naissance de la Confédération, il faut mentionner Joseph-Édouard Cauchon, journaliste et homme politique qui, en 1858 et en 1865, réunit des articles publiés sur le fédéralisme dans son Journal de Québec. ${ }^{24}$ Simple député, il prononça au cours du débat de l'hiver de 1865 un des bons discours en faveur de la Confédération.25

\section{LES HOMMES POLITIQUES ANGLAIS}

La Confédération a été surtout l'œuvre des hommes politiques des colonies britanniques, mais on ne peut oublier le rôle important qu'ont joué quelques représentants de la métropole.

Il faut placer au premier rang, lord Monck. Après avoir été député à la Chambre des communes et avoir fait partie de 1855 à $1857 \mathrm{du}$ gouvernement de lord Palmerston, il avait été nommé en 1861 gouverneur général de toutes les colonies britanniques en Amérique du Nord à une époque où Londres craignait les répercussions de la Guerre de Sécession. En juin 1864, il contribua à la formation du cabinet de coalition d'où sortit la

\footnotetext{
${ }^{24}$ Etude sur l'union projetée des provinces britanniques du Nord (Reproduite du "Journal de Québec"), (Québec, Typographie d'Auguste Côté et Cie, 1858) ; L'Union des provinces de l'Amérique britannique du Nord par l'hon. Joseph Cauchon (Extrait du "Journal de Québec"), (Québec, de l'Imprimerie A. Côté et Cie, 1865).

25 Débats, 559-590.
} 
Confédération. ${ }^{23}$ Pendant les années qui suivirent, il fit tout ce qui était en son possible pour favoriser la naissance du pays dont il devait devenir, en 1867, le premier gouverneur général. Il était en Angleterre au moment de la Conférence de Londres et lors des débats au Parlement britannique. Membre de la Chambre des lords, il prononça en faveur du projet un bref discours dans lequel il s'efforça de montrer que l'opposition de la Nouvelle-Écosse n'était pas sérieuse. ${ }^{27}$

Un artisan anglais important, à la fois politique et financier, de la naissance de la Confédération fut Edward William Watkin. En 1865, le chef des libéraux canadiens-français, Antoine-Aimé Dorion déclarait: "Les gens du Grand Tronc sont au fond de l'affaire (le projet de confédération), et je vois qu'à la dernière des actionnaires de la compagnie, M. Watkin les a d'avance félicités sur la brillante perspective qui s'ouvrait devant eux, par l'accroissement de valeurs qu'obtiendraient leurs actions et leurs bons, grâce à l'adoption du projet de confédération et à la construction de chemin de fer intercolonial comme partie de ce projet." 28 Tous comprenaient que le Watkin dont il était question était sir Edward Watkin qui était alors député à la Chambre des communes, mais qui était mieux connu comme financier intéressé au développement des chemins de fer. Il était venu une première fois en Amérique en 1851 et en 1861 il avait été envoyé au Canada par le duc de Newcastle, secrétaire d'état aux colonies afin d'enquêter sur les possibilités d'unir les colonies britanniques en un seul état. Dans un volume de souvenirs Canada and the States Recollection, 1851 to 1866, London, 1887, Watkin a peut-être exagéré son rôle, mais il est sûr que dans le but de favoriser les intérêts des chemins de fer et des financiers britanniques, il aida les Pères de la Confédération et surtout facilita leurs relations avec la métropole.

${ }^{26}$ Reginald S. Trotter, "Lord Monck and the Great Coalition of 1864", The Canadian Historical Review, 3 (1922) : 181-186.

27 Hansard's Parliamentary Debates. Third Series (London 1867), CLXXXV: 580 .

28 Débats parlementaires 1865, 225. 
En Grande-Bretagne même, quatre hommes ont été plus particulièrement liés au projet: le duc de Newcastle, Edward Cardwell, C. B. Adderley et lord Carnarvon.

Henry Clinton, duc de Newcastle, fut secrétaire d'état aux colonies dans le ministère "whig" de lord Palmerston, de 1859 à 1864. En 1864, il accompagna le prince de Galles, le futur Edouard VII, dans son voyage dans les colonies britanniques et il semble avoir alors compris que le fédéralisme était nécessaire pour les colonies. Quoi qu'il en soit, c'est Newcastle qui, le 6 juillet 1862, dans une dépêche au lieutenant-gouverneur de la Nouvelle-Écosse, Mulgrave, autorisa d'une façon générale les réunions qui devaient se terminer par la réalisation du fédéralisme. ${ }^{29}$ On a l'habitude de lui attribuer en grande partie l'attitude de la métropole, car ses successeurs ne firent que suivre l'orientation qu'il avait donnée. $\AA$ une époque où en GrandeBretagne quelques-uns souhaitaient se débarrasser des colonies en les laissant passer aux fítats-Unis, Newcastle crut à la solution du fédéralisme. ${ }^{30}$

Newcastle, dont la santé périclitait, fut remplacé comme secrétaire des colonies par Edwward Cardwell.

Protégé et ami de Robert Peel dans sa jeunesse, ayant derrière lui une longue carrière politique lorsqu'il devint secrétaire aux colonies dans le ministère de Palmerston, Cardwell fut un des premiers hommes politiques britanniques à comprendre que les colonies devaient être de plus en plus abandonnées à elles-mêmes et qu'elles devaient assumer davantage la responsabilité de leur défense. C'est ainsi que pour lui la réalisation de la Confédération canadienne s'inscrivit dans une vision générale d'un nouvel empire, celui que souhaitait un homme scandalisé qu'en Nouvelle-Zélande la guerre contre les Maoris ait coûté aussi cher aux contribuables britanniques. II

29 Cf. Pope's Confederation Documents, 303.

30 Reginald S. Trotter écrit: "Believing in colonial autonomy, he was eager at the same time to retain the colonies within the empire. The favourable change in the official British attitude towards the colonies was really due in large part to his influence." Canadian Federation in London (MCMXXI), V: 139. 
abandonna son poste lorsque le gouvernement de Palmerston fut, en 1866, remplacé par celui de lord Derby, mais il demeura un des plus ardents partisans de la Confédération canadienne. C'est pourquoi, le 28 février 1867, il prononça à la Chambre des communes un discours dans lequel il approuvait sans réticence la proposition du sous-secrétaire d'état aux colonies Adderley qui avait proposé le projet de l'Acte de l'Amérique du Nord britannique. ${ }^{31}$

Le successeur de Cardwell comme secrétaire d'état dans le ministère "tory" de lord Derby qui prit le pouvoir en 1867 fut lord Carnarvon, mais ce dernier siégeait à la Chambre des lords. Il eut comme sous-secrétaire d'état, à la Chambre des communes, Charles Bwyer Adderley. C'est ce dernier qui, à la Chambre basse, prononça le principal discours en faveur du projet. Sa tâche fut facile, appuyé qu'il fut par Cardwell. Il y eut bien un député du nom de John Bright qui rappela l'opposition de la Nouvelle-Écosse, mais en général le projet fut appuyé par les députés des deux partis et il fut adopté avec quelques amendements de forme. ${ }^{32}$

Il avait été approuvé auparavant à la Chambre des lords où il avait pris naissance et où siégeait le secrétaire d'état aux colonies, lord Carnarvon. Celui-ci prononça avant la deuxième lecture un discours qui est demeuré une des principales interprétations des intentions de ceux qui firent la Confédération canadienne. ${ }^{33}$ Carnarvon a été assez lié à notre histoire ainsi qu'en témoigne le recueil de ses discours concernant le Canada publié en 1902.34 Il vint en 1883 dans le pays qu'il avait con-

31 Hansard's Parliamentary Debates, Third Series (London, 1867), CLXXXV: 1174-1179. Sur Edward Cardwell, cf. Dictionary of National Biography, 3 (London, 1908): 952-954. 17-20.

32 Adderley, cf. The Dictionary of National Biography Supplement, 1:

33 Hansard's Parliamentary Debates, Third Series (London, 1867), CLXXXV: 558-576b.

34 Speeches on Canadian Affairs by Henry Howard Molyneux, Fourth Earl of Carnarvon, edited by Sir Robert Herbert, S.C.B. (London, John Murray, Alhermale Street, 1902). Carnarvon a été l'objet en 1925 d'une excellente biographie: The Life of Henry Molynewx Herbert, Fourth Earl of Carnarvon, by the Right Hon. Sir Arthur Hardinge, edited by Elizabeth, Countess of Carnarvon (Three vols., London: Oxford University Press, 1925), 441, 391, 400, 383 . 
tribué à créer. Il arriva, le ler septembre 1883, à Québec où il fut reçu comme un personnage illustre et fut l'hôte à la Citadelle du gouverneur le marquis de Lorne et de la princesse Louise. ${ }^{35}$

Tels ont été, rapidement évoqués, les hommes qui, au siècle dernier, ont contribué à créer le fédéralisme canadien. Il n'est pas facile d'établir une hiérarchie dans les mérites et les responsabilités, mais il semble bien que les vedettes qui se détachent du groupe sont George-Etienne Cartier, George Brown, John A. Macdonald et le duc de Newcastle. Sans les deux premiers, les querelles stériles des dernières années de l'Union auraient pu se poursuivre longtemps; sans Macdonald, la réalisation concrète du projet aurait été plus lente encore et sans Newcastle la métropole n'aurait pas compris qu'une solution était urgente. À ces noms, il faut ajouter ceux de Charles Tupper et Leonard Tilley, sans qui la Nouvelle-Écosse et le Nouveau-Brunswick auraient été encore plus difficiles à convaincre. En réalité, il n'y a qu'une poignée d'hommes qui méritent vraiment le titre de "Pères de la Confédération".36

35 Le Canadien, lundi 3 septembre 1883.

36 Sans vouloir pénétrer dans le domaine bibliographique qui a été assigné à $M$. Patrick Allen, j'ai donné quelques références que je voudrais compléter par de brèves remarques générales. Chaque Père de la Confédération et chaque personnage lié à la Confédération ont été l'objet de livres ou au moins d'articles de revue. La consultation du Macmillan Dictionary of Canadian Biography, préparé par William Stewart Wallace (Third edition, Toronto, 1963) et du Dictionary of National Biography de GrandeBretagne permet de trouver à chaque biographie la plupart des références quoique parfois, il en manque. Quelques ouvrages méritent toutefois une mention spéciale.

Il en est un tout d'abord dont le titre peut tromper, c'est Canadian Confederation and its leaders de M. O. Hammond (Toronto 1917). On y trouve dix-sept biographies assez élémentaires.

Deux Pères de la Confédération, John A. Macdonald et George Brown, ont eu l'honneur de deux excellentes biographies: John A. Macdonaid de Donald Creighton, I (Toronto, 1952); II (Toronto, 1955); Brown of the Globe de J. M. Careless, I (Toronto, 1959) ; II (Toronto, 1963).

Au sujet de Thomas d'Arcy McGee, on peut lire The Life of Thomas d'Arcy McGee, d'Isabel Skelton (Gardenvale, 1925), d'Alexander T. Galt, The Life and Times of Sir A. T. Galt, de O. D. Skelton (Toronto, 1920), et au sujet de Charles Tupper, The Life and Letters of the Rt. Hon. Sir Char- 
les Tupper, Bart, de E. M. Saunders (2 vols., London, 1916; supplement ed. de Sir C. H. Tupper, Toronto, 1926).

Il faut avouer que les meilleurs livres sur la naissance de la Confédération ont été écrits en anglais, et sur les quatre Pères de la Confédération canadiens-français, nous n'avons pas de bons ouvrages. Une étude scientifique et définitive de la vie et de l'œuvre de George-Etienne Cartier n'a pas encore été écrite, et il faut se contenter actuellement du Sir GoorgeEtienne Cartier, baronnet, publié par John Boyd (Montréal, 1918).

Sur Etienne-Paschal Taché, Hector Langevin, Antoine-Aimé Dorion, il n'y a que quelques articles de revues et sur Jean-Charles Chapais, on peut consulter le tome II des Mémoires Chapais de Julienne Barnard (Montréal. 1961).

JeAN-ChaRLes BONENFANT 